\title{
Electrochemiluminescence platform for the detection of C-Reactive Protein : Application of recombinant antibody technology to cardiac biomarker detection
}

\author{
Emmet J. O’Reillya,b, Paul J. Conroy ${ }^{\mathrm{b}, \mathrm{c}}$, Stephen Hearty ${ }^{\mathrm{b}}$, Tia E. Keyes ${ }^{\mathrm{b}}$, Richard \\ O’Kennedy ${ }^{\mathrm{b}}$, Robert J. Forster ${ }^{\mathrm{b}}$, Lynn Dennany ${ }^{\mathrm{c}}$
}

\begin{abstract}
This work exploits the high-affinity of recombinant antibodies and low background electrochemiluminescence (ECL) for cardiac-biomarker detection. The developed assay is capable of fg $\mathrm{mL}^{-1}$ detection limits as well as the detection of C-Reactive Protein (CRP) over a clinically relevant range. The assay demonstrated robust reproducibility, selectivity and stability while also highlighting a novel platform for detection of cardiac biomarkers at low concentrations.
\end{abstract}

\section{Introduction}

Sensitive detection and accurate analysis of protein biomarker molecules are absolutely essential for the early detection, treatment and management of many diseases. In addition the recognition of disease biomarker patterns has a significant role to play in future disease prevention.[1, 2] Current approaches to biomarker detection typically involve ELISA or sandwich type immunoassays coupled with fluorescent or electrochemical detection. In addition electrochemiluminescence (ECL) based systems such as that developed by Roche have been commercialised and are currently used by clinicians worldwide for the detection of protein biomarkers. Sandwich-type immunoassays for biomarker detection typically employ a capture antibody immobilised on a surface that selectively enriches the concentration of target antigen at the sensor surface. A labeled secondary antibody then binds to the captured antigen and facilitates quantitative measurement of the analyte concentration. Common issues with ELISA based assays include sensitivity and specificity issues primarily caused by non-specific in addition to sub-optimal antibody orientation on the surface. In addition the challenges of immobilising heavy chain antibodies of approximately $80 \mathrm{kDa}$ to biosensor transduction platforms also persist. Recent advances in antibody engineering have enabled the development of low molecular weight single chain fragment variable $(\mathrm{scFv})$ antibodies.[3, 4]. Primarily due to their reduced size and high specificity there has been much interest in

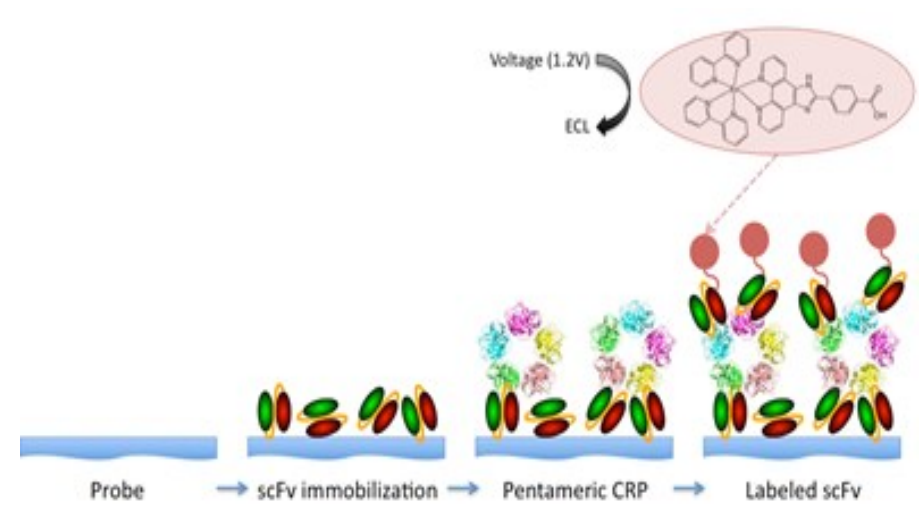

Scheme 1. Schematic illustration of the fabrication of recombinant antibody based biosensor for the detection of C-Reactive protein. The stepwise fabrication of the sensor is highlighted, $s c F v$ immobilization onto electrode or electrochemical probe, binding of pentameric CRP followed by binding of metal labeled ScFv fragments.

ScFv recombinant antibody technology and its potential application in the area of biomarker detection. [5] Despite being significantly smaller in size (typically $25 \mathrm{kDa}$ ) $\mathrm{scFv}$ recombinant antibodies retain the antigen specificity of their respective parent idiotypes. From an assay design perspective the resulting size reduction enables a significantly larger amount of the biological recognition element to be immobilised on a biosensor surface thereby reducing issues associated with non-specific binding and antibody surface orientation.[6] Moreover, recent developments in the fields of antibody libraries and expression bacteria have enabled recombinant antibodies to be engineered at lower cost and within a significantly shorter timeframe than those prepared in a living vertebrate host. Furthermore, recombinant antibodies such as $\mathrm{scFv}$ fragments contain peptide linkers between the VL and $\mathrm{VH}$ chains which can be easily functionalized to promote selfassembly on Pt and Au electrode surfaces. To date however, the application of recombinant antibody technology to the field of bio-sensing and specifically biomarker detection remains to be fully exploited. The work herein documents a novel electrochemiluminescent biosensor for the detection of $\mathrm{C}$ Reactive protein. The potential advantages of combining 
recombinant antibody technology with existing highly sensitive electrochemiluminescent detection are made clear. The relevance of such technology to cardiac biomarker detection is demonstrated by utilising the cardiac biomarker C-Reactive protein as the target analyte.

\section{Electrochemiluminescent detection of $\mathbf{C}$-Reactive protein}

CRP is an acute phase reactant in the inflammation response and one of a growing number of cardiac risk biomarkers that are used to determine a person's risk of developing cardiovascular disease (CVD). While CRP is an indicator of inflammation, its presence at elevated levels is associated with an increased risk of stroke and myocardial infarction,[7] therefore, its accurate detection is of significant importance to clinicians and medical practitioners alike. Current clinical testing methods suffer from a relative lack of sensitivity with cut off limits of $3-5 \mu \mathrm{g} \mathrm{mL}{ }^{-1}$. High Sensitivity CRP (hs-CRP) ELISA-based testing kits with sensitivities of $3 \mathrm{pg} \mathrm{mL}^{-1}$ are now commercially available (Abcam). As previously stated the high sensitivity and low background signals of ECL biosensors are well documented $[8,9]$ and it was therefore anticipated that the incorporation of the significantly smaller yet highly specific scFv antibody fragments into an ECL-based cardiac biosensor would enhance detection limits due to (a) a higher concentration of capture antibody on the electrode surface (b) more capture antibodies having the correct surface orientation and (c) a reduction in non-specific binding due to the absence of a full length antibody. In addition, ECL-based systems offer the ability to tailor ECL probe materials for multiple analyte detection. A schematic of the developed assay can be seen in Scheme 1. Recombinant $\mathrm{scFv}$ antibodies with high affinity for monomeric C-Reactive Protein (mCRP $K_{D}-3.53$ x 10 $\mathrm{M})$ [10] were purified and were then immobilised on a $\mathrm{Pt}$ electrode surface followed by absorption and subsequent capture of pentameric CRP (pCRP) in solution during a 20 minute timeframe. The high affinity of the $\mathrm{scFv}$ antibodies for $\mathrm{C}$-Reactive protein ensured binding of both sizable and minute concentrations of the analyte protein to the electrode surface. The bound pCRP was then detected by ECL using the same recombinant $\mathrm{scFv}$ antibody fragment covalently labelled with the luminescent metal probe $\left[\mathrm{Ru}\left(\text { bpy }{ }_{2} \mathrm{PICNH}_{2}\right]^{2+}\right.$. Labeling of the luminescent metal probe was examined by spectroscopy, with solutions of labeled antibody producing absorption and emission profiles typical of the ruthenium metal probe (Supplementary data, Figure S1). The ECL assay procedure was carried out as previously described using tripropylamine (TPA) as a co-reactant. The electrocatalytic oxidation of TPA by ruthenium metal centres is a common mechanism which can result in the production of an ECL response when the oxidised co-reactant (TPA) and the oxidised ruthenium metal centre of the label react.[11, 12] Figure 1 shows the dependence of ECL emission over a CRP concentration range $\left(5 \mathrm{fg} \mathrm{mL}^{-1}\right.$ to $600 \mathrm{ng}$ $\left.\mathrm{mL}^{-1}\right)$. The ECL emission observed at $1.2 \mathrm{~V}$ versus a silver/silver chloride $(\mathrm{Ag} / \mathrm{AgCl})$ reference electrode, corresponds with previous reports for a ruthenium metal centre and the shape of the curve is in accordance with ruthenium based ECL dynamics. The peak currents correspond to the electrocatalytic oxidation of TPA, (tri-n-propylamine) by the ruthenium label covalently attached to the anti-CRP $\mathrm{scFv}$ fragment. The absence of an ECL signal at $0.8 \mathrm{~V}$ illustrates that the ECL mechanism does not involve the "revisited route", where TPA radicals diffuse and react directly with $\left[\mathrm{Ru}(\mathrm{bpy})_{3}\right]^{2+}$ to generate the excited state. The ECL signal observed at $1.2 \mathrm{~V}$ vs. $\mathrm{Ag} / \mathrm{AgCl}$ is exclusive of the oxidation potential of TPA.

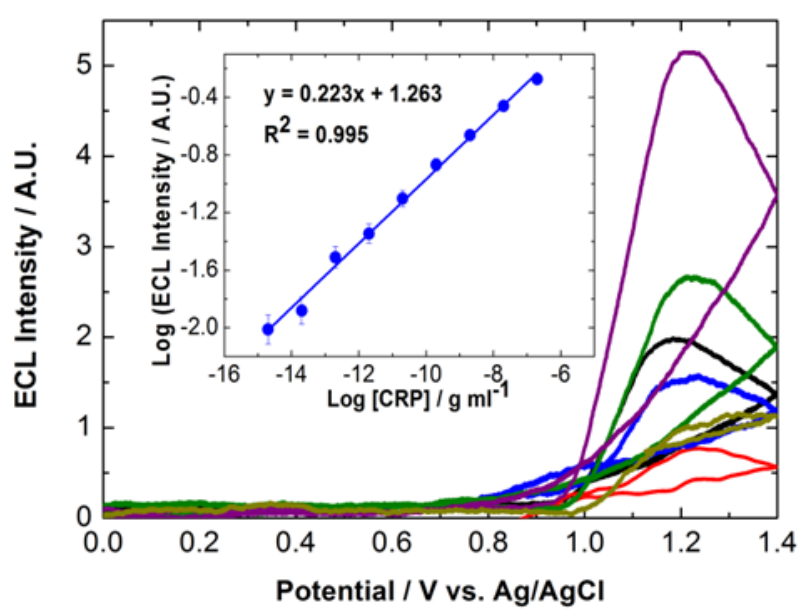

Figure 1. Dependence of the ECL emission intensity on the CRP concentration. From top to bottom at $+1.2 \mathrm{~V}$, the

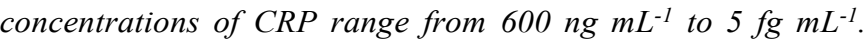
Not all ECL responses are shown. The inset shows dependence of the logarithm of the maximum ECL intensity on log[CRP]. The error bars are comparable to, or smaller than, the size of the symbols

The distance between adjacent redox sites is a key factor governing the efficiency of the electron-transfer processes. It is equivalent to the charge diffusion in redox polymers involving an electronhopping process from site to site where transport of both electrons and charge compensating counter ions occurs simultaneously.[11, 13] The rate of electron transfer $\mathrm{k}$ decays exponentially with the distance $r$ between redox centres in the transition state. A bimolecular rate of electron transfer for $\mathrm{Ru}^{2+*}$ to $\mathrm{Ru}^{3+}$ of $\sim 10^{6} \mathrm{M}^{-1} \mathrm{~s}^{-1}$ was reported previously, highlighting the fact that the excited state can be quenched efficiently by $\mathrm{Ru}^{3+}$ species in close proximity to the excited state $\mathrm{Ru}^{2+*} .[13,14]$ Although electron-hopping may be possible between adjacent ruthenium centres, given the extremely low levels of label present on the electrodes due to the low levels of antigen present, it is unlikely that the assay is affected by this phenomena. ECL production, however, is highly dependent upon the rate of charge transfer $\left(\mathrm{D}_{\mathrm{CT}}\right)$. A fast rate of charge transfer ensures sufficient $\mathrm{Ru}^{3+}$ will be present to react with a given analyte and produce ECL. An insufficient amount of $\mathrm{Ru}^{3+}$ will result in decreased ECL emission and hence decreased sensitivity. The $\mathrm{D}_{\mathrm{CT}}$ value for the $\left[\mathrm{Ru}(\mathrm{bpy})_{2} \mathrm{PICH}_{2}\right]^{2+}$ is typical of $\mathrm{Ru}^{2+/ 3+}$ systems, yielding a value of $\sim 10^{9} \mathrm{~cm}^{-1} \mathrm{~s}^{-1}$ consistent with previously published work. $[10,-12-14]$ This value suggests that despite the minute concentrations of ruthenium label present, charge transfer via 
diffusion is not a limiting factor for the assay thereby further highlighting the suitability of ECL as a transduction mechanism for this assay. The voltammetric behavior of $\left[\mathrm{Ru}(\text { bpy })_{2} \mathrm{PICH}_{2}\right]^{2+}$ and the Randles-Sevçik plots are supplied in the supplementary material, Figure S2.

A wide variety of hsCRP assays are currently available commercially with linear ranges from $0.1 \mathrm{mg}$ to $10 \mathrm{mg} / \mathrm{L}$, and as such are sufficiently sensitive to measure the vast majority of patients presenting at a clinic. As such the bio-sensor herein was developed not as a replacement for such systems but to demonstrate the potential advantages of combining $\mathrm{scFv}$ antibody technology with ECL from the perspective of sensitivity, cost of production, response time and robustness. Figure 1 (inset), shows the dependence of the logarithm of the maximum ECL intensity on $\log [\mathrm{CRP}]$ over a clinically significant range. The maximum value of $600 \mathrm{ng} \mathrm{mL}-1$ corresponds to $0.6 \mathrm{mg} \mathrm{L}^{-1}$, which is within the clinical range for low risk levels of CRP. Of significantly more interest is the lower limits of detection possible with this approach. The analytical sensitivity or limit of detection (LOD) was calculated using the formula [Average ECL emission of the blank + 3SD (blank)]. The LOD for the assay was calculated to be $0.3 \mathrm{fg} \mathrm{mL}$ 1 considerably lower than the $\mathrm{pg} \mathrm{mL}^{-1}$ detection limits of current commercially available CRP testing kits or the $\mathrm{ng} \mathrm{ml}^{-1}$ LODs obtained by electrochemical detection[14, 15]. The limit of quantification (LOQ) for the assay was calculated to be approximately $2 \mathrm{fg} \mathrm{mL}-1$. While the clinical relevance of these figures may be limited to CRP concentrations typically occurring at pg $\mathrm{mL}^{-1}$ levels, if applied correctly the approach has the potential to improve current techniques for the monitoring of other myocardial infarction biomarkers such as Troponin $\mathrm{T}$ which are currently undetectable by conventional assays at sub $10 \mathrm{pg} \mathrm{mL}^{-1}$ concentrations. It is for applications such as this that the authors envisage the newly developed assay offers the most potential from a clinical perspective. While sensitivity is of immense importance in biosensor design, its significance is surpassed only by selectivity. While antibody/antigen interactions possess a high degree of specificity this is often negated by an inaccurate transduction process, often leading to the production of a false positive. To further validate the new strategy's utility and specificity a series of controls were designed and analyzed experimentally. Figure 2 shows the ECL response obtained for incomplete variants of the sandwich assay. The ECL signal circa $1.2 \mathrm{~V}$ was only observed when the complete sandwich assay was tested and indicates that a positive result is only observed in the presence of C-reactive protein. To assess variability parameters of the assay the results were performed using a sample size of $n=3$ on $n=3$ days. Figure 3 shows the averaged ECL response for 3 separate assays conducted in three consecutive days.

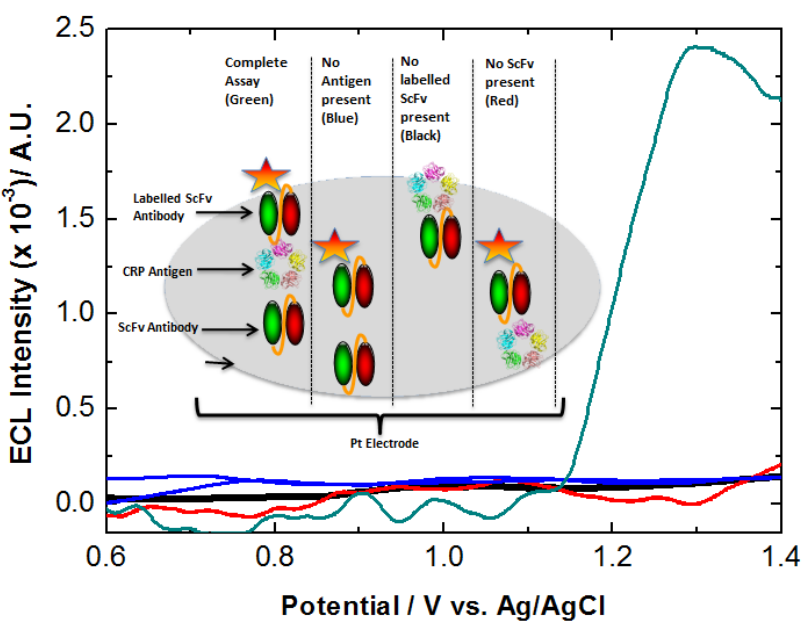

Figure 2. Dependence of the ECL emission intensity for modified film, without the scFv CRP antibody (red), without CRP antigenprotein (blue), without labeled $S c F v$ CRP antibody (black) and with the complete sandwich assay (green). Inset shows a schematic of each of these controls. The CRP concentration used for the complete sandwich assay corresponds to $50 \mathrm{pg} \mathrm{mL}^{-1}$.

In each case the CRP antigen concentration was $5 \mathrm{fg} \mathrm{mL}^{-1}$. Given the low quantity of analyte present some variability in the detection signal was expected, however, the associated standard deviation was calculated to be $\sim 7 \times 10^{-5}$ indicating excellent inter-electrode reproducibility. To assess the long term stability of the sensor ECL measurements were performed over 21 days (S3 supplementary material). During this timeframe an overall reduction in ECL intensity of $4.8 \%$ was recorded, corresponding favourably with previously reported stability data for $\mathrm{Ru}(\mathrm{bpy}) 3^{2+}$ based ECL sensing platforms.[16, 17] As expected the ECL response time for the sensor excluding CRP incubation times varies with potential scan rate. A scan rate of $0.1 \mathrm{mVs}^{-1}$ results in a response time of approximately 56 seconds.

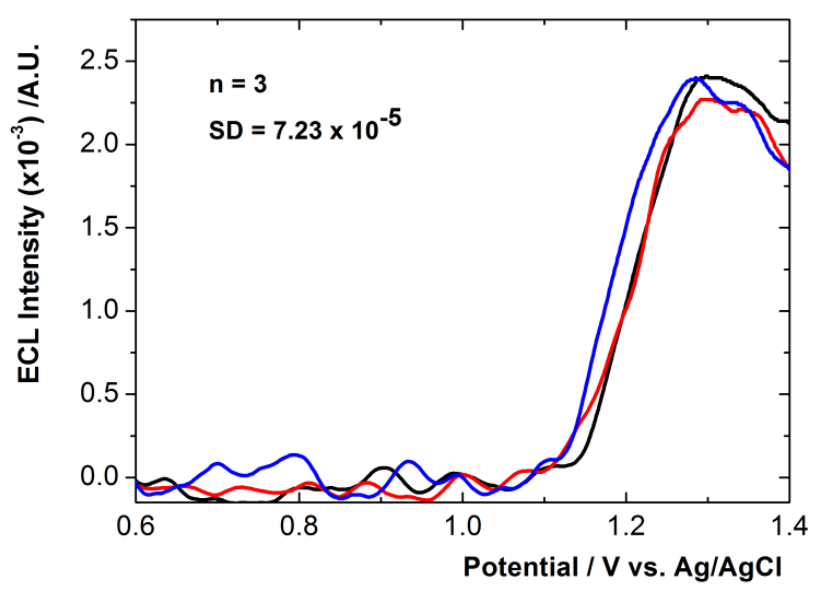

Figure 3. The averaged ECL response for 3 different assays

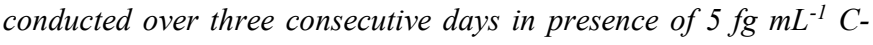
Reactive protein. 
Previous work has shown that these $\mathrm{scFv}$ recombinant antibodies are extremely efficient at selectively discerning specific CRP antigens from other competing antigens.[18] Although not examined directly within this study, this previous work highlights the utility of this assay in selectively detecting CRP over the biological relevant concentration range. Due to the inherent nature of ECL no photonic excitation source is required thereby resulting in a significant cost saving and reduced background interference in comparison to UV and traditional fluorescent based detection systems. In addition, the lower costs associated with the production of recombinant antibodies compared to traditional antibody production methods involving vertebrate hosts ensure that the developed sensor is a promising platform for future point of care health monitoring.

\section{Conclusions}

In summary, this work highlights a proof-of-concept example for a new, robust and feasible ECL sensor for the determination of CRP with high sensitivity. We have combined recombinant antibody technology with ECL for the first time to produce a powerful assay for the determination of the cardiac biomarker $\mathrm{C}$-Reactive protein at the fg level, which to our knowledge is the first reported sensor capable of detecting CRP at these detection levels. More importantly, this example paves the way for a broader application of recombinant antibody engineering across other biologically relevant biomarkers and we are currently pursuing this possibility. The authors anticipate that in combining recombinant antibody engineering with low background detection techniques such as ECL, there exists much potential for the development of a new generation of sensitive, selective and rapid biosensor as well a significant scope for the improvement of existing systems, all with a common goal of improving point-of-care health monitoring.

\section{Notes}

a Materials and Surface Science Institute, Department of Chemical and Environmental Science, University of Limerick, Castletroy, Limerick, Ireland

$b$ Biomedical Diagnostics Institute, National Centre for Sensor Research, Dublin City University, Dublin 9, Ireland

c Current address: Department of Biochemistry and Molecular Biology, Faculty of Medicine, Nursing and Health Science, Monash University, Melbourne, VIC 3800, Australia.

d WestCHEM, Department of Pure and Applied Chemistry, Thomas Graham Building, University of Strathclyde, Glasgow, G1 $1 X L, U K$

Electronic Supplementary Information (ESI) available: This includes spectroscopic and electrochemical data for $\left[\mathrm{Ru}(\mathrm{bpy})_{2} \mathrm{PICH}_{2}\right]^{2+}$. [xxxxxx]. See DOI: $10.1039 / \mathrm{c} 000000 \mathrm{x} /$

\section{Corresponding Author}

emmet.oreilly@ul.ie

lynn.dennany@strath.ac.uk

\section{Author Contributions}

EOR, LD and RF conceived the idea and designed the experiments. PJC and ROK engineered the recombinant antibody fragments. EOR, PJC and LD interpreted the data and contributed to the writing of the manuscript.

\section{Acknowledgements}

The authors acknowledge financial support from the Marie Curie Reintegration Grant (PIRG-2010-268236), Science Foundation Ireland (SFI), under CSET grant number 10/CE/B1821 (PJC, SH, ROK, RF, TK, EOR) and the Irish Research Council for Science, Engineering and Technology (IRCSET) Embark scholarship (PJC).

\section{References}

1. McDonnell, B., et al., Cardiac biomarkers and the case for point-ofcare testing. Clinical Biochemistry, 2009. 42(7-8): p. 549-561.

2. Eriksson, S., S. Wittfooth, and K. Pettersson, Present and future biochemical markers for detection of acute coronary syndrome. Crit Rev Clin Lab Sci, 2006. 43(5-6): p. 427-95.

3. Borrebaeck, C.A. and C. Wingren, Recombinant antibodies for the generation of antibody arrays. Methods Mol Biol, 2011. 785: p. 247-62.

4. Holliger, P. and P.J. Hudson, Engineered antibody fragments and the rise of single domains. Nat Biotechnol, 2005. 23(9): p. 112636.

5. Zeng, X.Q., Z.H. Shen, and R. Mernaugh, Recombinant antibodies and their use in biosensors. Analytical and Bioanalytical Chemistry, 2012. 402(10): p. 3027-3038.

6. Gilbert, I., et al., Double chip protein arrays using recombinant single-chain Fv antibody fragments. PROTEOMICS, 2004. 4(5): p. 1417-1420.

7. Conroy, P.J., et al., Antibody production, design and use for biosensor-based applications. Seminars in Cell \& Developmental Biology, 2009. 20(1): p. 10-26.

8. Dennany, L., R.J. Forster, and J.F. Rusling, Simultaneous direct electrochemiluminescence and catalytic voltammetry detection of DNA in ultrathin films. Journal of the American Chemical Society, 2003. 125(17): p. 5213-5218.

9. Forster, R.J., P. Bertoncello, and T.E. Keyes, Electrogenerated Chemiluminescence. Annual Review of Analytical Chemistry, 2009. 2: p. 359-385.

10. Leonard, P., et al., High throughput ranking of recombinant avian $s c F v$ antibody fragments from crude lysates using the Biacore A100. Journal of Immunological Methods, 2007. 323(2): p. 172-179.

11. O'Reilly, E.J., et al., Insights into electrochemiluminescent enhancement through electrode surface modification. Analyst, 2013. 138(2): p. 677-82.

12. Venkatanarayanan, A., et al., Ruthenium aminophenanthroline metallopolymer films electropolymerized from an ionic liquid: Deposition and electrochemical and photonic properties. Langmuir, 2008. 24(19): p. 11233-11238. 
13. O'Reilly, E.J., et al., Ground and excited state communication within a ruthenium containing benzimidazole metallopolymer. Phys Chem Chem Phys, 2011. 13(15): p. 7095-101.

14. Fakanya, W.M. and I.E. Tothill, Detection of the Inflammation Biomarker C-Reactive Protein in Serum Samples: Towards an Optimal Biosensor Formula. Biosensors, 2014. 4(4): p. 340357.

15. Bryan, T., et al., An optimised electrochemical biosensor for the label-free detection of C-reactive protein in blood. Biosensors and Bioelectronics, 2013. 39(1): p. 94-98.

16. Hun, X. and Z. Zhang, A Novel Electrogenerated Chemiluminescence (ECL) Sensor Based on Ru(bpy)32+-Doped Titania Nanoparticles Dispersed in Nafion on Glassy Carbon Electrode. Electroanalysis, 2008. 20(8): p. 874-880.

17. Zhou, C., et al., Electrospun Ru(bpy)32+-doped nafion nanofibers for electrochemiluminescence sensing. Analyst, 2010. 135(5): p. 1004-1009.

18. McDonnell, B., Development of novel antibody-based diagnostics for the early and rapid detection of cardiac markers, Thesis Dissertation - Retrieved from http://doras.dcu.ie/15710/. 